\title{
Anthracene aquacracking using $\mathrm{NiMo} / \mathrm{SiO}_{2}$ catalysts in supercritical water conditions
}

\author{
T.R. Reina ${ }^{1}$, P.Yeletsky ${ }^{2,3}$, J.M. Bermúdez ${ }^{1}$, P. Arcelus-Arrillaga ${ }^{1}$, V.A. Yakovlev ${ }^{2,3,4} *$, \\ M.Millan ${ }^{1 *}$ \\ ${ }^{1}$ Department of Chemical Engineering, Imperial College London London SW7 2AZ, UK \\ ${ }^{2}$ BoreskovInstitute of Catalysis, Lavrentieva Ave. 5, Novosibirsk, Russian Federation \\ ${ }^{3}$ UNICAT LLC, Lavrentieva Ave. 5, Novosibirsk, Russian Federation \\ ${ }^{4}$ Novosibirsk State University, Pirogova St. 2, Novosibirsk, Russian Federation \\ *Corresponding authors: marcos.millan@imperial.ac.uk, yakovlev@catalysis.ru
}

\begin{abstract}
A series of effective $\mathrm{NiMo} / \mathrm{SiO}_{2}$ catalysts for heavy oil upgrading in supercritical water have been developed. Experimental results with anthracene as model compound resembling structures present in heavy oils showed that the catalytic activity as well as the liquid and gas product distributions are governed by catalyst composition. In particular by adjusting the Ni/Mo ratio different physicochemical properties (crystalline phase composition, particle size and catalysts reducibility) are obtained, which have influence on catalytic behavior. A variety of liquid products together with a valuable gas (rich in $\mathrm{H}_{2}$ ) are produced in this process, which takes place with remarkably low coke deposition on the catalysts. Overall, the results derived from this work confirm the viability of upgrading polyaromatic structures in supercritical water using Ni-Mo catalysts and provides an insight on the main parameters to control in catalyst design.
\end{abstract}

Keywords: anthracene oxidation, heavy oil upgrading, supercritical water, Ni-Mo catalysts, hydrogen. 


\section{Introduction}

As conventional oil resources are dwindling and their reserves are less easily accessible [1], the production of heavy crude oil ( $\mathrm{HCO}$ ) has become more relevant and will remain so in the foreseeable future [2]. In this sense, there is a need for more efficient refining processes to transform HCO into lighter fuels. Conventional processes for increasing the value of heavy oil fractions aim to increase the $\mathrm{H} / \mathrm{C}$ ratio of fuel, generating lighter fractions. However, this implies either rejecting a large amount of the carbon in the feed, as in thermal and catalytic cracking processes, or using high pressure hydrogen, an expensive gas, in hydrocracking processes [3].

The use of $\mathrm{H}_{2} \mathrm{O}$ instead of $\mathrm{H}_{2}$ in the so called steam cracking has been proposed by several groups as a potential way to upgrade heavy and unconventional oils [4-8]. Hydrothermal heavy oil upgrading has shown important improvements compared to thermal upgrading methods as higher yields to lighter liquid product fractions are obtained at the expense of a reduction in the amount of coke produced [9, 10]. Moreover, high degree of metal [11, 12] and heteroatom removal $[13,14]$ were achieved in hydrothermal heavy oil upgrading processes. It was suggested that in traditional upgrading processes, coke formation proceeds through the propagation reactions of radicals beginning at an active site on the catalyst and the reactor walls [15]. The nature and mechanism of coke formation is greatly affected by the presence of water, which produces a highly porous solid compared to the one obtained through neat pyrolysis [1]. Different methods have been developed to further suppress coke formation in hydrothermal heavy oil upgrading processes. One such method is increasing hydrogen provision to system, which can be achieved by including additives, adding an oxygen source to carry out partial oxidation followed by water gas shift to produce hydrogen in situ with an adequate catalyst [16]. 
The implementation of homogeneous or heterogeneous catalysts in hydrothermal processes like chemical synthesis, waste destruction and biomass conversion in near critical water (NCW) and supercritical water (SCW) has been extensively considered and reviewed [17, 18]. Water becomes a supercritical fluid when it reaches a temperature of $374{ }^{\circ} \mathrm{C}$ or above, a pressure of 221 bar or above and a density of $0.32 \mathrm{~g} / \mathrm{ml}$. At these conditions water properties as a solvent change drastically from a polar to a non-polar solvent [19]. One of the main challenges for the implementation of catalytic processes in NCW and SCW is to find materials that are catalytically active and sufficiently stable at these conditions [20]. For instance, Fumoto et al. developed a series of iron-zirconium mixed oxide catalysts effective in converting petroleum residual oils into lighter fuels [21]. Later on, the same group introduced $\mathrm{Al}_{2} \mathrm{O}_{3}$ in the catalyst formulations aiming to avoid $\mathrm{FeO}_{\mathrm{x}}$ sintering and thus improving the material stability [22]. The use of noble metal catalysts such as $\mathrm{Pt}, \mathrm{Pd}, \mathrm{Ru}$, and $\mathrm{Rh}$ on a support with adequate surface activity has been reported to exhibit overall good performance [23]. A different study on the desulfurization of heavy oil in SCW considered the use of more economical catalysts like $\mathrm{ZnO}, \mathrm{MoO}_{3}$ and $\mathrm{MoS}_{2}$. They observed that the presence of the catalysts improved the desulfurization capacity of the system. Analysis post reaction showed that $\mathrm{MoS}_{2}$ remain unchanged during reaction while $\mathrm{ZnO}$ and $\mathrm{MoO}_{3}$ suffered morphological changes related to sulfidation reactions [13, 24].

As an alternative, catalytic cracking in supercritical water (SCW) conditions using metal based catalysts represents a fairly new approach to upgrade heavy oils. This method takes advantage of the above mentioned properties of SCW. Under these conditions, aromatic rings are well dissolved and dispersed in the reaction medium, favoring the contact between reactants and catalyst particles. Additionally, the upgrading process in SCW typically takes place with significantly low coke formation [9]. 
The chemistry of heavy oils is complex, making it difficult to obtain a thorough comprehension of the reactions taking place. Hence, studies with model compounds representing chemical structures found in heavy oils can provide relevant information about the reactivity of different structures, role of the catalysts and most likely reaction pathways, as recently shown using anthracene as a model compound in subcritical steam cracking reactions [7].

Formulations based on Ni-Mo on a support providing the cracking function remain an attractive catalyst for HCO upgrading. Many factors like the preparation method, support choice and Ni/Mo ratio may affect the activity of Ni-Mo supported catalysts [26]. In particular, given the fundamental role played by the metals in this type of reaction, the Ni/Mo ratio can be considered a key parameter in the catalyst design. In this scenario, this work aimed to develop a series of $\mathrm{NiMo} / \mathrm{SiO}_{2}$ catalysts with different $\mathrm{Ni} / \mathrm{Mo}$ ratios for anthracene upgrading in SCW conditions. The correlation of the physicochemical characterization of the samples with the exhibited catalytic behavior was analyzed in order to identify the most relevant catalyst features required to maximize upgrading performance.

\section{Experimental}

\subsection{Catalyst preparation}

The catalysts were prepared by sol-gel method described elsewhere [27]. The desired amounts of powdered commercial $\mathrm{NiCO}_{3} \cdot \mathrm{mNi}(\mathrm{OH})_{2} \cdot \mathrm{nH}_{2} \mathrm{O}$ and $\mathrm{MoO}_{3}$ were mixed with the required amount of aqueous ammonia solution $\left(25 \% \mathrm{NH}_{3}\right)$ in deionized water in a $100 \mathrm{~mL}$ beaker under continuous stirring at $1000 \mathrm{rpm}$ for $1 \mathrm{~h}$. Then the appropriate quantity of tetraethyl orthosilicate (TEOS) was added. After $1 \mathrm{~h}$, the mixture was heated up to $100{ }^{\circ} \mathrm{C}$ and kept at this temperature during $1 \mathrm{~h}$ under continuous stirring. Then, the mixture was dried in an oven for complete water 
and ethanol evaporation at $100{ }^{\circ} \mathrm{C}$ overnight. Afterwards, the mixture was calcined at $500{ }^{\circ} \mathrm{C}$ for $1 \mathrm{~h}$ and finally, the catalysts were partially reduced in $\mathrm{H}_{2}$ flow at $550{ }^{\circ} \mathrm{C}$ for $1 \mathrm{~h}$ followed by passivation with ethanol.

\subsection{Catalyst characterization}

X-ray diffraction (XRD) studies were performed on a D8 diffractometer (Bruker, Germany) using the monochromatic $\mathrm{CuK} \alpha$ radiation $(\lambda=0.1542 \mathrm{~nm})$. The mean size of the coherentscattering domain (CSD) was determined based on the broadening of the reflections using the Selyakov-Scherrer equation.

Temperature programmed reduction (TPR) analysis of the $\mathrm{NiMo} / \mathrm{SiO}_{2}$ catalyst series were carried out with the catalysts initially in a completely oxidized form. A gas mixture of $\mathrm{H}_{2}(10 \%)$ and $\operatorname{Ar}(90 \%)$ at a flow rate of $20 \mathrm{~mL} \cdot \mathrm{min}^{-1}$ was used in these studies. $0.1 \mathrm{~g}$ of catalyst was placed in a U-shaped quartz reactor and heated in a reducing atmosphere with constant heating rate of $4{ }^{\circ} \mathrm{C} \min ^{-1}$ up to $900{ }^{\circ} \mathrm{C}$. Changes of $\mathrm{H}_{2}$ concentration in the gas mixture at the exit of the reactor were measured with a thermal conductivity detector (TCD).

Textural characteristics of the prepared samples were obtained from the isotherms of nitrogen adsorption measured at $-196{ }^{\circ} \mathrm{C}$ by means of an automatic volumetric device ASAP 2400 (Micromeritics, USA). The samples were pre-treated at $150{ }^{\circ} \mathrm{C}$ under residual pressure of $c a$. 102 torr. The values of the total pore volume and the BET surface area $\left(\mathrm{A}_{\mathrm{BET}}\right)$ were calculated from the adsorption isotherms under relative pressures $\mathrm{P} / \mathrm{P}_{0}\left(\mathrm{P}_{0}\right.$ is the saturation pressure $)$ of 0.98 and $0.05-0.2$, correspondingly. The volume of micropores $\left(\mathrm{V}_{\mu}\right)$ accessible to nitrogen at $77 \mathrm{~K}$ and the total surface area of mesopores and macropores were determined according to 
comparative method in a form reported by Karnaukhov et al. [28], which is analogous to the $\alpha_{s^{-}}$ method by Sing and $t$-method by Lippens-de Boer [28, 29].

Transmission electron microscopy analysis (TEM) was performed on a JEM-2010 (JEOL, Japan) electron microscope with a lattice resolution of $0.14 \mathrm{~nm}$ and an accelerating voltage of $200 \mathrm{kV}$. The samples for the TEM study were prepared by ultrasonic dispersing in ethanol and consequent deposition of the suspension upon a "holey" carbon film supported on an aluminum grid. Local elemental analysis was performed with the EDX method on an energy-dispersive Xray Phoenix spectrometer equipped with a $\mathrm{Si}(\mathrm{Li})$ detector with an energy resolution of $130 \mathrm{eV}$.

The carbon content in the spent catalysts has been obtained from CHNS-O analysis performed by means of an Elemental Vario EL Cube (Elementar Analysensysteme GmbH, Germany) CHNS-O analyzer. All analyses were carried out by triplicate.

\subsection{Catalytic Activity}

Anthracene aquacraking experiments were performed in a stainless steel microbomb batch reactor with a volume of $18 \mathrm{~mL}$. The operation of the reactor has been described elsewhere [30]. The reactor was filled with $0.2 \mathrm{~g}$ of anthracene $98 \%$ (Sigma-Aldrich), $0.2 \mathrm{~g}$ of catalyst and the volume of water required to reach 230 bar pressure at $425{ }^{\circ} \mathrm{C}(3.9 \mathrm{~mL})$. The air trapped in the reactor was purged with He to ensure an oxygen-free atmosphere.

After the desired reaction time (60 min), the reactor was quenched to stop the reaction. Reaction products were recovered with a chloroform:methanol mixture in 4:1 (v/v) ratio. The catalyst was recovered by filtration. The amount of coke deposited onto the catalyst was determined by HCNS-O analysis. The liquid sample was analyzed in a Perkin Elmer Clarus 500 gas chromatograph (GC) fitted with a flame ionization detector (FID). The GC was equipped with a 
non-polar HT-5 $(25 \mathrm{~m} \times 0.32 \mathrm{~mm})$ column from SGE, UK. The products were identified in a Varian Star 3400/Saturn 2000 GC-MS by comparison with the spectrum found in the instrument library. The GC-MS was fitted with the same column as the GC-FID and operated using the same temperature program. Quantification of reaction products was performed by establishing GC response factors of anthracene and the main polyaromatic products, by injection of samples with known concentrations prepared using standards.

Gas products were analyzed in a Perkin Elmer Clarus 500 GC equipped with thermal conductivity detector (TCD). The GC was equipped with a capillary Carboxen 1010 Plot (30 m $\times$ $0.53 \mathrm{~mm}$ ) column for analysis of $\mathrm{H}_{2}, \mathrm{CH}_{4}, \mathrm{CO}$ and $\mathrm{CO}_{2}$. The TCD was periodically calibrated with a certified calibration gas mixture.

Yields to gas, liquid and solid (coke) products ( $\mathrm{Y}_{\mathrm{gas}}, \mathrm{Y}_{\mathrm{LP}}$ and $\mathrm{Y}_{\text {coke }}$ respectively) are defined as the percentage of carbon moles transferred from the original anthracene to the gas, liquid and solid fractions obtained. They are calculated by dividing the amount of carbon moles in each fraction by the total carbon moles of the anthracene loaded to the reactor (Equations 1-3). Finally, selectivity to liquid products $\left(\mathrm{S}_{\mathrm{LP}}\right)$ is defined as the percentage of the carbon moles of the reacted anthracene that are transferred to the liquid products and is calculated by dividing the amount of carbon moles in the liquid fraction between the amounts of carbon moles corresponding to the anthracene reacted (Equation 4). The carbon mass balance of the products was closed typically within a $\pm 5 \%$ experimental error.

$$
\begin{aligned}
& Y_{\text {gas }}=\frac{C_{\mathrm{CH}_{4}+C_{\mathrm{CO}}+C_{\mathrm{CO}_{2}}}}{C_{\text {anthracene fed }}} \\
& Y_{L P}=\frac{C_{\text {benzophenone }}+C_{\text {xanthene }}+C_{\text {xanthone }}+C_{\text {fluorenone }}+C_{\text {anthraquinone }}}{C_{\text {anthracene fed }}}
\end{aligned}
$$


$Y_{\text {coke }}=\frac{C_{\text {coke }}}{C_{\text {anthracene fed }}}$

Equation 3

$S_{L P}=\frac{C_{\text {benzophenone }}+C_{\text {xanthene }}+C_{\text {xanthone }}+C_{\text {fluorenone }}+C_{\text {anthraquinone }}}{C_{\text {anthracene reacted }}}$

Equation 4

Where $\mathrm{C}_{\mathrm{CH}_{4},}, \mathrm{C}_{\mathrm{CO}_{2}}$ and $\mathrm{C}_{\mathrm{CO}}$ represent carbon moles of each of these gases in the gas fraction, $\mathrm{C}_{\text {benzophenone }}, \mathrm{C}_{\text {xanthene, }}, \mathrm{C}_{\text {xanthone, }}, \mathrm{C}_{\text {fluorenone }}$ and $\mathrm{C}_{\text {anthraquinone }}$ represent the carbon moles of each of the main liquid products and $\mathrm{C}_{\text {anthracene fed }}$ and $\mathrm{C}_{\text {anthracene reacted }}$ represent the carbon moles of anthracene in the feed and converted to the different products, respectively.

\section{Results and discussion}

The nominal compositions of the catalysts (assuming $\mathrm{Ni}$ and $\mathrm{Mo}$ in the reduced metallic state) together with their textural properties are presented in Table 1. All the samples are mesoporous materials with specific surface area and porosity governed by the Ni/Mo ratio. Particularly, the specific surface area decreases when molybdenum loading increases. Both average pore diameter and pore volume present a maximum for an intermediate Ni/Mo composition (Ni20Mo60) and are slightly lower for the two extreme formulations (Ni40Mo40 and Ni10Mo70).

Table 1 Nominal composition (wt. \%) assuming metallic state for Ni and Mo and main textural properties of the catalysts prepared.

\begin{tabular}{ccccccc}
\hline Catalyst & $\begin{array}{c}\text { Ni } \\
(\mathbf{w t .} \%)\end{array}$ & $\begin{array}{c}\text { Mo } \\
(\mathbf{w t .} \%)\end{array}$ & $\begin{array}{c}\mathbf{S i O}_{\mathbf{2}} \\
(\mathbf{w t . \%})\end{array}$ & $\begin{array}{c}\mathbf{A}_{\text {BET }} \\
\left(\mathbf{m}^{\mathbf{2}} \cdot \mathbf{g}^{-\mathbf{1}}\right)\end{array}$ & $\begin{array}{c}\mathbf{V}_{\text {Pore }} \\
\left(\mathbf{c m}^{\mathbf{3}} \cdot \mathbf{g}^{\mathbf{- 1}}\right)\end{array}$ & $\begin{array}{c}\mathbf{D}_{\text {pore }} \\
(\mathbf{n m})\end{array}$ \\
\hline Ni40Mo40 & 40 & 40 & 20 & 87 & 0.17 & 8.1 \\
Ni30Mo50 & 30 & 50 & 20 & 74 & 0.24 & 13.0 \\
Ni20Mo60 & 20 & 60 & 20 & 68 & 0.25 & 14.9 \\
Ni10Mo70 & 10 & 70 & 20 & 44 & 0.12 & 10.7 \\
\hline
\end{tabular}


The XRD patterns of the studied samples are presented in Figure 1. Several noteworthy differences can be extracted from the diffractograms. For example, in the case of Ni40Mo40 some reflexions ascribed to metallic Ni particles were detected at $2 \theta=44.0$ and $51.1^{\circ}$ JCPDS card $\left(\mathrm{N}^{\mathrm{o}} 45-1027\right)$. These peaks are slightly shifted $\left(0.3^{\circ}\right)$ pointing to some degree of Mo incorporation in the $\mathrm{Ni}$ lattice resulting in the formation of Ni-Mo solid solutions and/or intermetallide compounds. For instance, the observed reflexions resemble those of the $\mathrm{MoNi}_{4}$ intermetallide species (PDF $\mathrm{N}^{\mathrm{o}}$ 65-5480 ICSD). The mean particle size of this type of Ni-Mo particles was $8 \mathrm{~nm}$ (estimated using Scherrer equation). Peaks corresponding to $\mathrm{NiO}$ were not detected, indicating complete reduction of the $\mathrm{Ni}$ species after pretreatment.

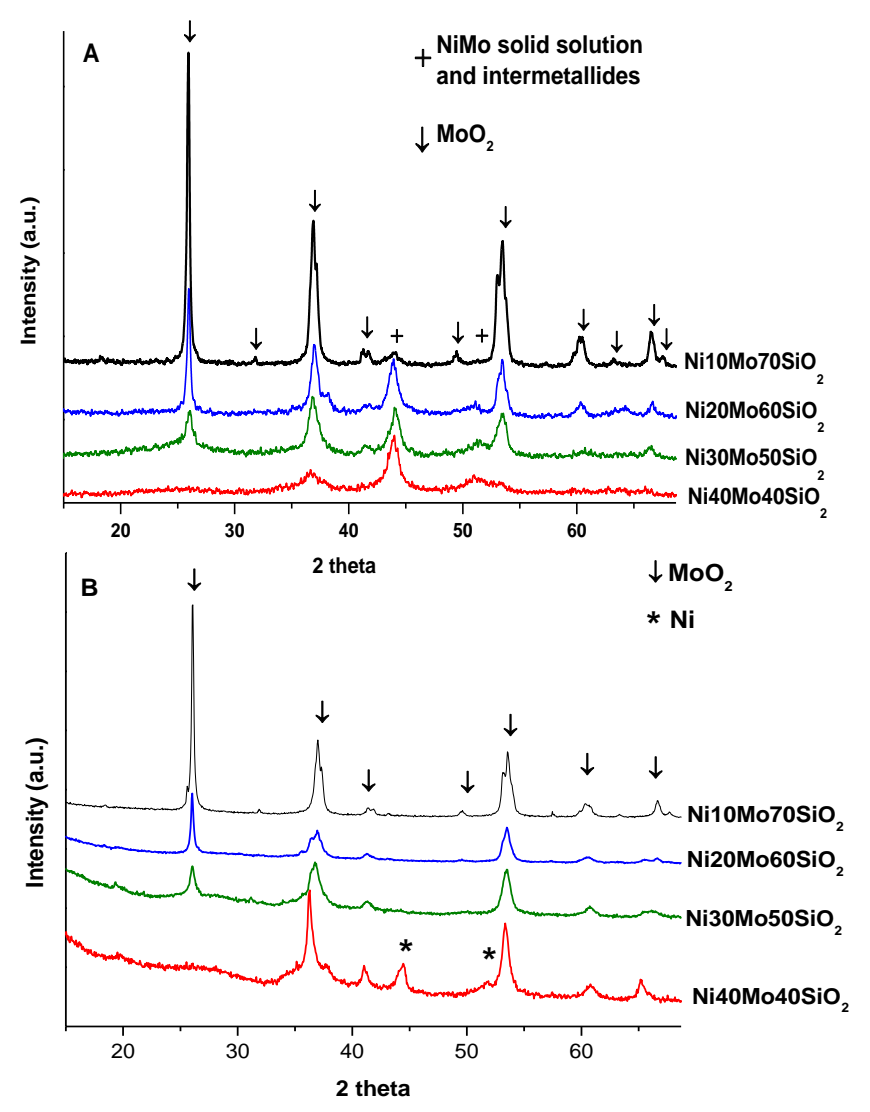

Figure 1. X-ray diffraction patterns of the prepared catalysts. A) Fresh reduced samples; B) spent catalysts 
Remarkable changes in the diffractograms were observed upon increasing Mo loading. Growing peaks of $\mathrm{MoO}_{2}$ phases appear, indicating certain degree of molybdenum oxide segregation in the form of Tugarinovite (PDF $\mathrm{N}^{\mathrm{o}}$ 32-671). In addition, the peaks related to Ni species lose intensity and finally disappear at high Mo contents. In the limit which corresponds to the Ni10Mo70 sample, the XRD pattern almost completely corresponds to $\mathrm{MoO}_{2}$ (peaks at $2 \theta=26.0,31.8,37.0$, $41.4,49.5,53.5,60.2,64.0$ and $\left.66.7^{\circ}\right)$. The mean particle size of the CSD for the Ni10Mo70 sample amounted to $c a .34 \mathrm{~nm}$. Even though the XRD patterns for the Ni10Mo70, Ni20Mo60 and Ni30Mo50 samples mainly show a $\mathrm{MoO}_{2}$ phase, other species, for example small and/or amorphous $\mathrm{Ni}$ nanoparticles, may be present in the solids. Moreover, different types of molybdenum phases should not be discarded. For instance, the small peak in the diffractogram of the Ni20Mo60 at $2 \theta=38.2^{\circ}$ may be attributed to $\mathrm{MoO}_{2}$ in a different crystalline phase form (PDF N $50-739)$.

On the other hand, Figure 1B shows the XRD patterns of the spent catalysts. Indeed, the stability of the catalysts in SCW is one of the major challenges for this technology and it is worth studying possible structural changes during the reaction. As shown in the figure, all the catalysts conserve their main crystalline features evidencing the stability of these materials under the reaction conditions. However, a careful analysis of the XRD patterns reveals some structural modifications. Firstly, a certain degree of $\mathrm{MoO}_{2}$ segregation was observed (this issue is more notable in the 404020 sample when the fresh and spent patterns are compared). Secondly, as consequence of $\mathrm{MoO}_{2}$ segregation, the peak at 2 theta $\sim 44.0$ (accounting Ni-Mo solid solution or intermetallide compounds) in the spent samples disappears. Nevertheless, peaks attributed to metallic Ni (JCPDS 4-850) with relatively small particle size are observed in the 404020 sample indicating that after the reaction the catalysts still conserve their main composition. The latter 
validates the role of $\mathrm{SiO}_{2}$ as textural promoter avoiding metal particles sintering. In addition, the presence of nickel oxidized species could not be fully discarded since some of the XRD peaks of these species could overlap with those of $\mathrm{MoO}_{2}$. Overall, the observed structural stability of prepared materials is already an achievement given the problematic in terms of stability displayed by other reference catalysts based on alumina or zeolites in supercritical water [23].

Further information regarding catalyst composition, particle sizes and species formed was obtained from transmission electron microscopy measurements. In view of the TEM study, the samples could be divided in two groups according to the similarities they present: Ni40Mo40Ni30Mo50 and Ni20Mo60-Ni10Mo70. Therefore, selected representative images of both types of catalysts are presented in Figures 2 and 3. Low magnification pictures of the Ni40Mo40 sample reveal a system mainly composed by roundish particles of approximately $1 \mu \mathrm{m}$. At higher magnification, it is seen that there are particles of Ni-Mo intermetallides and/or $\mathrm{MoO}_{2}$ phases identified by planar distances showed in Figure $2 c$ with the particles sizes ranging in the area of $1-20 \mathrm{~nm}$ as shown in Figure 2b. EDX spectrum (Figure $2 d$ ) of the cluster in the red circle (Figure 2a) confirms that the particles are composed of nickel and molybdenum phases pointing to an intimate Ni-Mo contact, in good agreement with the XRD data. Furthermore, the identified planar distance of $0.32 \mathrm{~nm}$ in the higher magnification image corresponds to the binary $\mathrm{Ni}_{3} \mathrm{Mo}$ compound. By contrast, the Ni10Mo70 sample mainly consists of large particles of lamellar $\mathrm{MoO}_{2}$, most probably decorated with some Ni particles (Figure 3). Families of planes with typical planar distance of intermetallic Ni-Mo compounds were not detected. On the images, mainly particles of $\mathrm{MoO}_{2}$ phase are observed. These particles present a porous structure forming a canal system. According to the EDX data neither $\mathrm{Ni}$ nor $\mathrm{Si}$ are incorporated in the large molybdenum oxide particles indicating a weaker Ni-Mo interaction. 

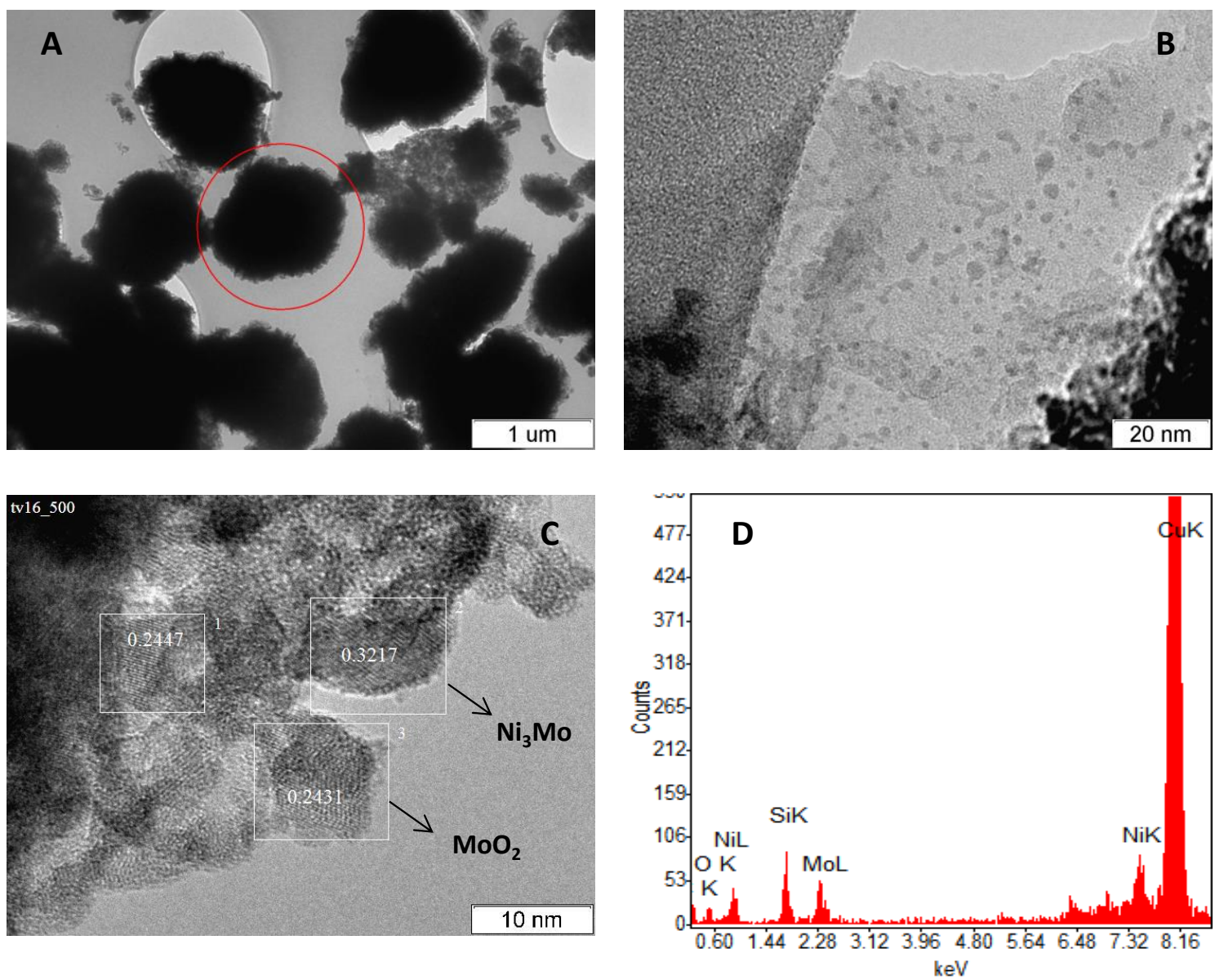

Figure 2. Selected TEM images of the Ni40Mo40 sample. Images $a, b$ and $c$ represent low, medium and high magnifications respectively while image $d$ is the typical EDX spectra of the particles in picture $a$ (particle surrounded with the red circle). 

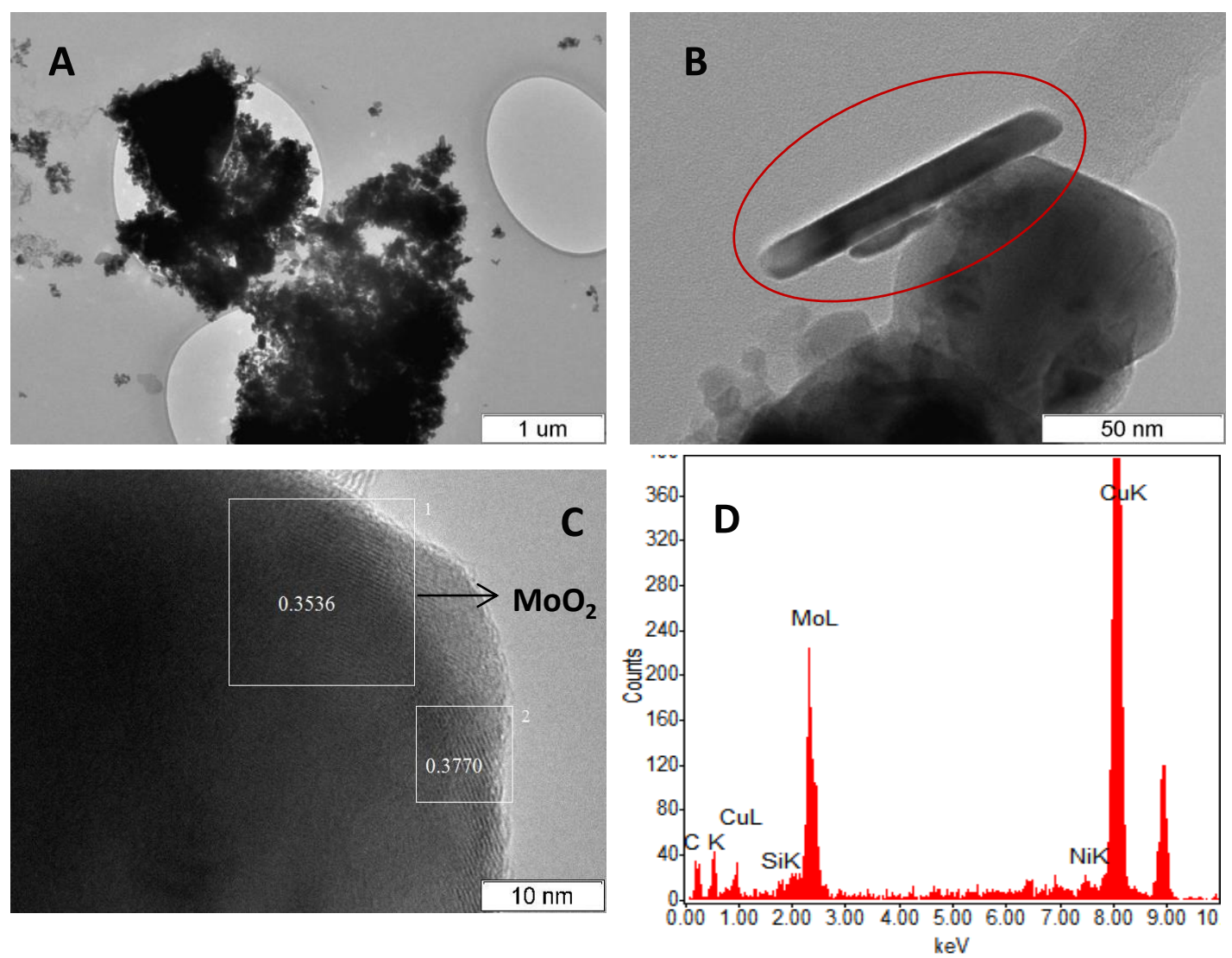

Figure 3. Selected TEM images of the Ni10Mo70 sample. Images $a, b$ and $c$ represent low, medium and high magnifications respectively while image $d$ is the EDX spectra of the large particle in picture $b$.

As partial oxidation of the aromatic rings, leading to $\mathrm{C}-\mathrm{O}$ bonds that are easier to crack than $\mathrm{C}-\mathrm{C}$ double bonds, can be significant in an upgrading process in SCW, the redox properties of the catalysts must be a relevant factor. Indeed, some interesting features can be extracted from the $\mathrm{H}_{2}$-TPR (Figure 4). As shown in the plot, all the catalysts present a complex profile involving the simultaneous reduction of $\mathrm{NiO}$ to $\mathrm{Ni}$ and several reduction steps of $\mathrm{MoO}_{3}$. The reduction profile of Ni40Mo40, the catalyst with the lowest Mo content of this set, exhibits three peaks shifted to the lower temperature region. This is actually the only sample presenting a relatively low temperature reduction process (peak at $415{ }^{\circ} \mathrm{C}$ ), which most likely corresponds to the 
reduction of $\mathrm{NiO}$ species in contact with molybdenum, as previously reported for Ni-Mo based catalysts [31]. However, the formation of mixed compounds due to the interaction with $\mathrm{SiO}_{2}$ and $\mathrm{MoO}_{3}$ such as nickel silicates and nickel molybdates and their reduction should not be discarded. The other two reduction zones at $537{ }^{\circ} \mathrm{C}$ and $700-850{ }^{\circ} \mathrm{C}$ are associated to different $\mathrm{MoO}_{3}$ reduction steps. More precisely, the low-temperature peak can be assigned to the partial reduction $\left(\mathrm{Mo}^{6+} \rightarrow \mathrm{Mo}^{4+}\right)$ of amorphous, highly defective, multilayered Mo oxides or heteropolymolybdates (octahedral Mo species) [31-33]. The high-temperature zone comprises the deep reduction of all Mo species, including highly dispersed tetrahedral Mo species. The peak at $712{ }^{\circ} \mathrm{C}$ could be related to crystalline phases of orthorhombic $\mathrm{MoO}_{3}$ with intermediate reducibility [34-36]. As for the Ni30Mo50 sample, a similar TPR profile was obtained although excluding the lower temperature peak at $c a .400{ }^{\circ} \mathrm{C}$. Molybdenum reduction in this sample takes place in the same temperature window as Ni40Mo40.

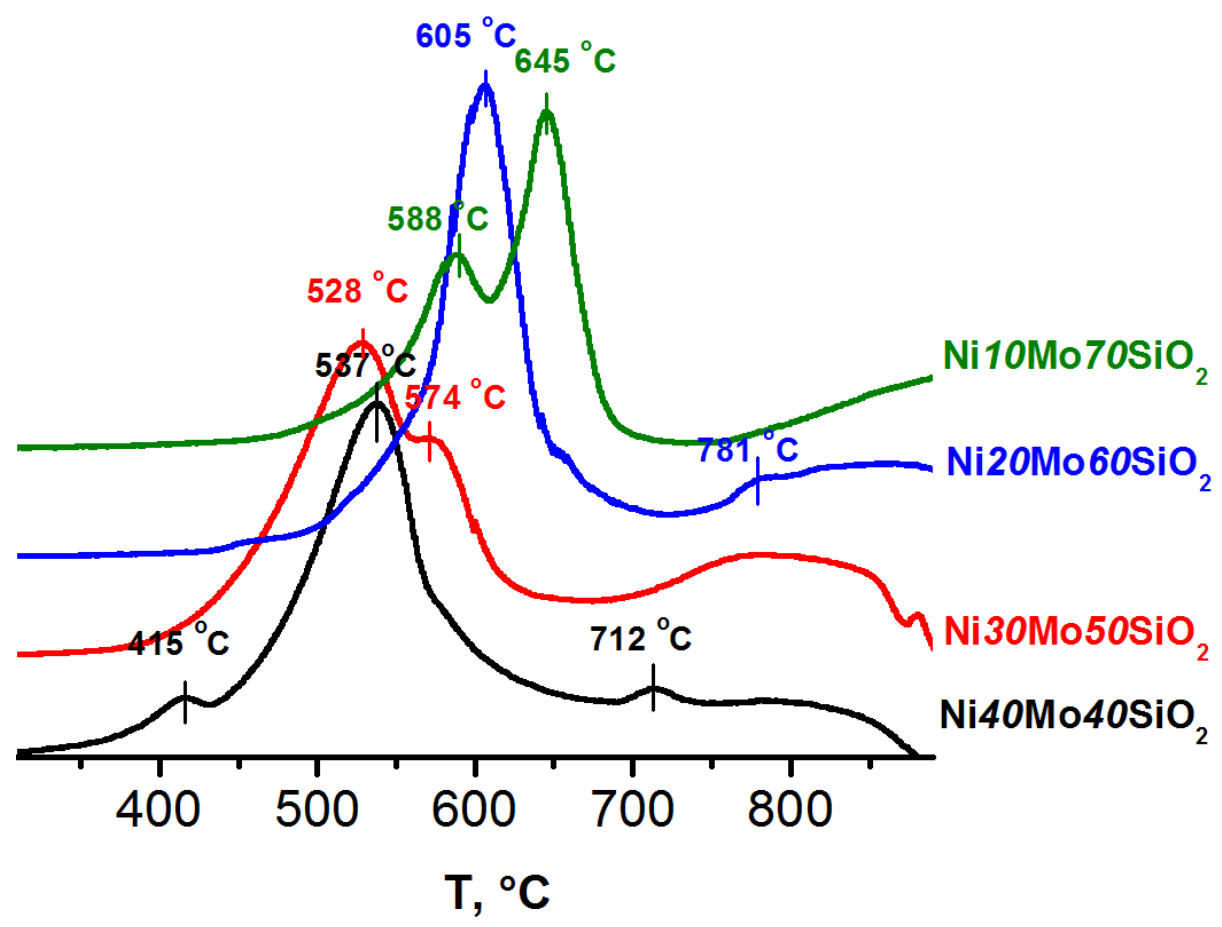

Figure 4. $\mathrm{H}_{2}$-TPR profiles of the studied catalysts. 
Nevertheless, TPR curves notably change for the catalysts with higher Mo loadings. The reducibility profiles of Ni20Mo60 and Ni10Mo70 are significantly different to those described above. All peaks are shifted towards higher temperature indicating a more difficult reduction. This is presumably the result of two effects: firstly, the lower amount of $\mathrm{Ni}$, which promotes the overall catalyst reducibility as observed for Ni40Mo40 and Ni30Mo30; secondly, the presence of larger molybdenum oxide particles (as suggested in the XRD discussion), which are more difficult to reduce. As a result, neither Ni20Mo60 nor Ni10Mo70 reached full reduction at 900 ${ }^{\circ} \mathrm{C}$.

In summary, based on TPR and XRD data it can be established that samples with higher Ni loadings (Ni40Mo40 and Ni30Mo50) consist of Ni-Mo ensembles with small particle size and well dispersed on the $\mathrm{SiO}_{2}$ matrix. On the other hand, while the samples with lower Ni quantities are composed by segregated molybdenum oxide particles with larger particle sizes. These $\mathrm{MoO}_{2}$ particles present weaker contact with Ni particles since no incorporation of Ni in the Mo matrix was detected. These features led to significant differences in redox behavior. Ni40Mo40 and Ni30Mo50 samples achieved complete reduction in the TPR experiment indicating that these solids present greater oxygen mobility compared to the others. In part, these enhanced redox properties arise from the Ni-Mo synergy, by which Ni acted as a promoter of Mo reducibility.

All these physicochemical properties had influence on catalytic behavior. Table 2 summarizes the conversions and the product yields achieved with these catalysts. As shown in Table 2, all the samples are active in the partial oxidation of anthracene. Conversions obtained after one hour followed the order: Ni40Mo40 $>$ Ni20Mo60 $\approx$ Ni30Mo50 $>$ Ni10Mo70, which suggests that Ni is essential to achieve good catalytic performance. The sample poorer in $\mathrm{Ni}$ was the least active while the richer in Ni resulted in higher conversions, with the two other samples presenting an 
intermediate behavior. The observed activity trend correlates well with the chemical properties of the samples discussed above. Ni40Mo40 sample is the material with the smaller Ni-Mo particle size providing more exposed metallic surface, which may enhance the adsorption and subsequent oxidation of the hydrocarbons. Furthermore, this sample shows better reducibility than the others and therefore it has greater oxygen mobility, an essential characteristic to catalyze an oxidation process.

Table 2 Anthracene conversion, carbon yields (C-mol\%) in liquid products, gas and coke together with liquid selectivity (C-mol\%) and hydrogen yield (L at normal conditions per gram of anthracene) at $425^{\circ} \mathrm{C}, 230 \mathrm{bar}$ and $1 \mathrm{~h}$ of reaction.

\begin{tabular}{ccccccc}
\hline Catalyst & $\begin{array}{c}\text { Conversion } \\
(\boldsymbol{\%})\end{array}$ & $\begin{array}{c}\mathbf{Y}_{\mathbf{L P}} \\
(\mathbf{\%})\end{array}$ & $\begin{array}{c}\mathbf{Y}_{\text {coke }} \\
(\boldsymbol{\%})\end{array}$ & $\begin{array}{c}\mathbf{Y}_{\text {gas }} \\
(\boldsymbol{\%})\end{array}$ & $\begin{array}{c}\mathbf{S}_{\mathbf{L P}} \\
(\boldsymbol{\%})\end{array}$ & $\begin{array}{c}\mathbf{H}_{\mathbf{2}} \\
(\mathbf{L} / \mathbf{g} \text { ant. })\end{array}$ \\
\hline Ni40Mo40 & 46.7 & 22.7 & 0.4 & 23.6 & 48.6 & 0.3 \\
Ni30Mo50 & 33.9 & 16.5 & 1.9 & 15.5 & 48.5 & 0.1 \\
Ni20Mo60 & 34.8 & 7.5 & 2.4 & 24.9 & 21.3 & 0.3 \\
Ni10Mo70 & 17.3 & 7.1 & 0.3 & 9.9 & 41.1 & 0.1 \\
\hline
\end{tabular}

Liquid $\left(\mathrm{Y}_{\mathrm{LP}}\right)$, coke $\left(\mathrm{Y}_{\text {coke }}\right)$ and gas yields $\left(\mathrm{Y}_{\mathrm{gas}}\right)$ also depend on the catalyst composition. The presence of $\mathrm{Ni}$ seems to favor liquid yields and liquid selectivity $\left(\mathrm{S}_{\mathrm{LP}}\right)$, as observed in experiments with Ni40Mo40 and Ni30Mo50 catalysts. By contrast, the gas fraction seems to be associated to Mo since the proportion of gas in the overall conversion is higher for the higher Mo loadings as seen in Ni20Mo60 and Ni10Mo70. Alternatively, it could also be associated to the lower Ni content which can stabilize the liquid products. In this sense, poor Ni loadings may potentiate the cracking properties of $\mathrm{SiO}_{2}$ leading to larger amounts of gas.

Interestingly, coke yields are remarkably low in all cases, which results advantageous in comparison with the traditional hydrocracking process where coke deposition is one of the main 
problems as it causes catalyst deactivation [9]. For instance, the coke yield in Ni40Mo40 is only $0.4 \%$, which is a promising result. Low yields to coke obtained seem to be related to SCW as reaction medium, which can aid in the suppression of coke formation and facilitates the dispersion of coke precursors as has been reported elsewhere [9, 37, 38].

Not only the catalytic activity and the product yields but also the liquid and gas product distributions are governed by the Ni/Mo ratio. Figure 5A shows distribution of the main liquid products formed in the process and Figure 5B presents their chemical structures. It was observed that flourenone, xanthene and benzophenone are the dominant products for catalysts with high $\mathrm{Ni}$ loadings while some different products, for example anthraquinone and xanthone, appear for the Ni10Mo70 sample. In all cases a diversity of liquid products from oxidation, ring opening and hydrogenation were obtained. Most of these liquids are oxygenated products obtained via partial oxidation of the original anthracene. It should be noted that water is not a strong oxidant and frequently this type of oxidation requires the presence of stronger oxidative agents such as hydrogen peroxide [39]. However, these $\mathrm{NiMo} / \mathrm{SiO}_{2}$ catalysts facilitate the partial oxidation of the polyaromatic compounds using just water as a reaction media. Furthermore, the presence of some compounds as benzophenone and flourenone involves $\mathrm{C}-\mathrm{C}$ bond breaking, needed in industrial upgrading of $\mathrm{HCO}$. 

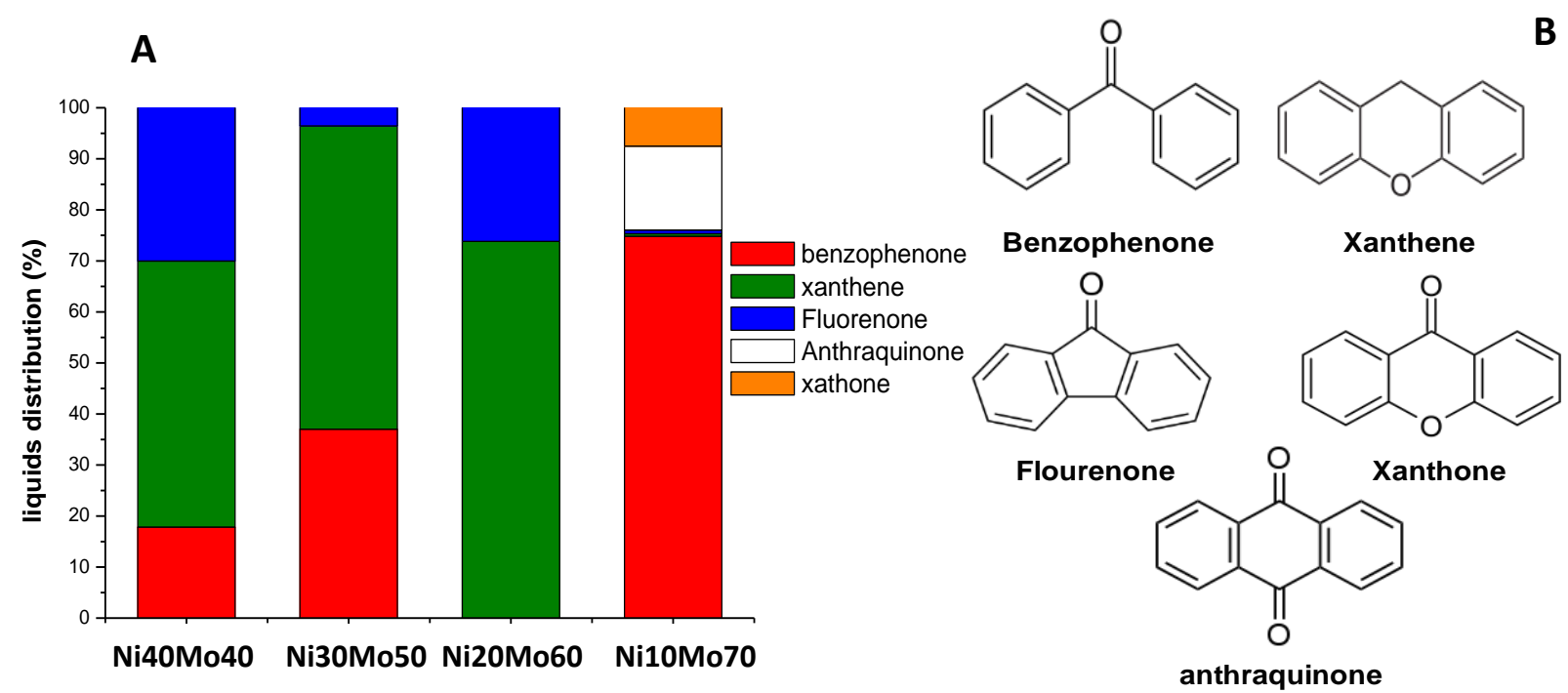

Figure 5. A) Liquids products distribution B) Chemical structure of the main identified liquids products.

The oxidation process is likely to start with the formation of $\mathrm{C}=\mathrm{O}$ bonds (partial oxidation) breaking the aromaticity of the central ring, which then becomes more reactive and prone to cracking. The central ring preferential oxidation that takes places in the aquacracking treating is noteworthy because it increases the atom efficiency of the HCO upgrading process compared to traditional hydrotreating processes, which predominantly proceeds from the external rings [39]. This difference in the atom efficiency of both processes is explained in Figure 6. Therefore, the oxygenated products observed in this study can be considered as early stage reaction products that may evolve to smaller molecules by cracking, carbon rejection and deoxygenation. 

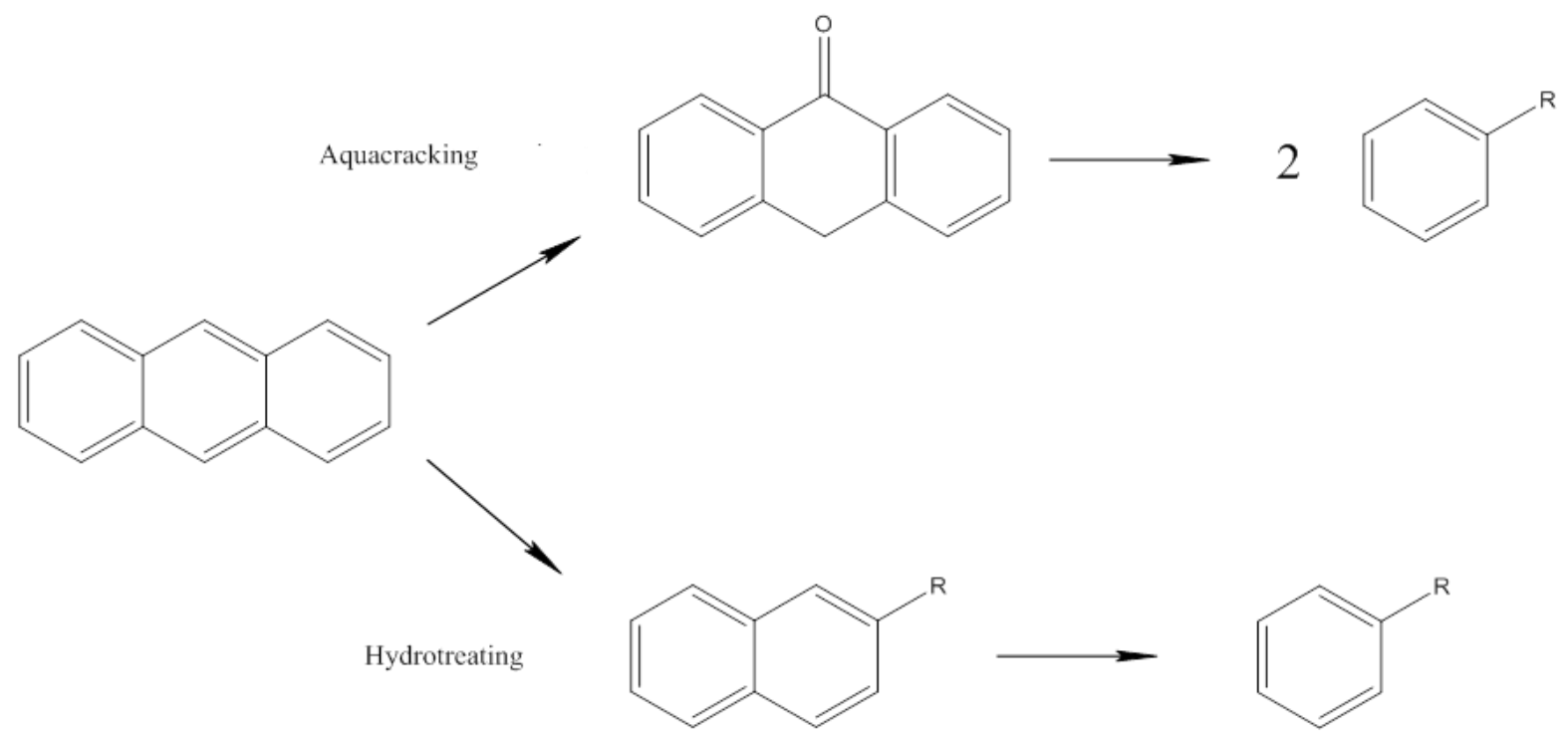

Figure 6. Schematic comparison of atom efficiency in anthracene aquacracking and conventional hydrocracking processes.

This reaction pathway would fit with a scheme proposed by DeCoster et al. for partial oxidation of vaporized anthracene [40]. As shown in the scheme (Figure 7), anthracene can be first oxidized to anthrone. However, anthrone is an unstable intermediate and was not detected in the liquid products in this study. It could have been further oxidized in a quick step to anthraquinone or may have evolved to fluorenone via carbon rejection. A combined carbon rejection and oxidation process transforms anthraquinone into xanthone, which can later evolve towards xanthene losing oxygen, in a reduction step that may involve $\mathrm{H}_{2}$ formed in the process. Xanthone is an oxidation product that also involves hydrogenation of the ring. It seems that in this upgrading process hydrogen generated in-situ is incorporated to the aromatic rings. Indeed, Zhao et al. also indicated that SCW could act as a hydrogen source transferring hydrogen via hydroxyl radical [41]. On the other hand, benzophenone very likely comes from the rupture of the C-C bond in fluorenone, although it can be also formed from anthraquinone losing a carbonyl group. 
As indicated in Figure 7, benzophenone, fluorenone and xanthene can be considered advanced products of the reaction that appear at late reaction stages, while anthraquinone is an initial reaction product. This is in good agreement with the catalytic activity observed and the liquid product distributions obtained. The most active samples presented considerable amounts of late reaction products (benzophenone, fluorenone and xanthene), while the least active material Ni10Mo70 presented a broader liquid product distribution, including relatively large amounts of anthraquinone, in agreement with its poorer activity.

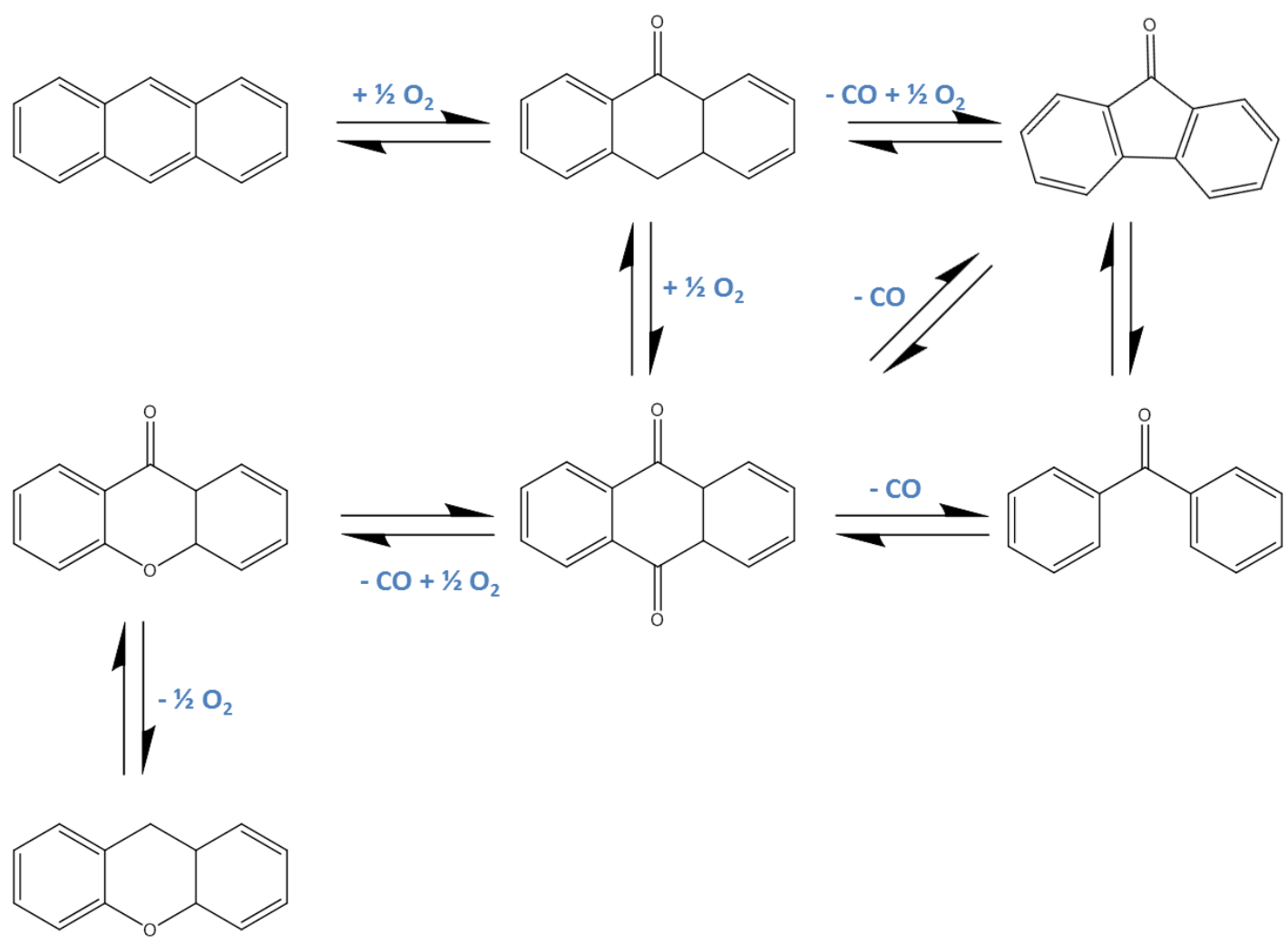

Figure 7. Proposed reaction pathway for the partial oxidation of anthracene in supercritical water conditions. (1) anthracene (2) anthrone (3) fluorenone (4) xanthone (5) anthraquinone (6) benzophenone (7) xanthene. 
Regarding the gas product distribution (Table 3), $\mathrm{H}_{2}$ was the most abundant gas produced in the reaction, irrespective of the catalysts composition, which is a positive result from the energy and economic point of view. The calculation of the volume of hydrogen produced per mass of anthracene loaded in the reactor showed that up to $0.3 \mathrm{~L}\left(\mathrm{~g}_{\text {anthracene }}\right)^{-1}$ can be produced in SCW conditions (Table 2). Together with $\mathrm{H}_{2}, \mathrm{CO}, \mathrm{CO}_{2}$ and $\mathrm{CH}_{4}$ were produced. Again, as observed for the liquid fraction, the gas product distribution varies with catalyst composition. In fact, simultaneous reactions such as water gas shift, reforming, $\mathrm{CO}$ and $\mathrm{CO}_{2}$ methanation are plausible since $\mathrm{Ni}$ is a typical active phase for them [42-44] and may have had influence on the gases obtained. In particular the relatively high hydrogen concentrations observed may have resulted from the excess of water in the media favoring reforming and shift reactions in addition to the capacity of the catalysts to promote water splitting. Furthermore, the observed partial oxidation of anthracene followed by cracking reactions led to the $\mathrm{CO}$ and $\mathrm{CO}_{2}$ presence in the gas phase.

Table 3 Gas product distribution (vol. \%) obtained in experiments at $425^{\circ} \mathrm{C}, 230$ bar and $1 \mathrm{~h}$ of reaction.

\begin{tabular}{ccccc}
\hline Catalyst & $\mathbf{H}_{\mathbf{2}}$ & $\mathbf{C O}$ & $\mathbf{C O}_{\mathbf{2}}$ & $\mathbf{C H}_{\mathbf{4}}$ \\
\hline Ni40Mo40 & 71 & 19 & 9.2 & 0.8 \\
Ni30Mo50 & 65 & 16 & 17.8 & 1.2 \\
Ni20Mo60 & 68 & 21 & 8.5 & 2.5 \\
Ni10Mo70 & 78.6 & 2.1 & 5.8 & 13.5 \\
\hline
\end{tabular}

\section{Conclusions}

A series of $\mathrm{NiMo} / \mathrm{SiO}_{2}$ catalysts has been tested in the anthracene aquacraking in SCW conditions. The samples present different structural and redox properties depending on the $\mathrm{Ni} / \mathrm{Mo}$ ratio. Higher catalytic activities are obtained when small Ni-Mo particles are in close contact and no segregation of $\mathrm{MoO}_{2}$ is attained. This favorable situation occurs at higher Ni 
loadings, which lead to the formation of Ni-Mo mixed compounds with good metallic dispersion and enhanced reducibility.

A variety of liquid products was obtained, including ring opening, oxygenated and hydrogenated species, confirming the process effectiveness in anthracene upgrading. Anthracene oxidation seems to preferentially proceed through the middle ring, which can potentially lead to higher atom efficiency than conventional upgrading processes such as hydrocracking. Anthraquinone is likely to play an intermediate role in the reaction pathway, being quickly produced by the oxidation of anthracene and reacting to form a number of reduction and cracking products.

A valuable gas stream (rich in $\mathrm{H}_{2}$ ) resulted from the process. This study shows that both liquid and gas product distributions strongly depend on Ni/Mo ratio, which seems to be a key parameter in catalyst design in order to optimize the overall performance.

\section{Acknowledgements}

This research was performed under the UNIHEAT project. The authors wish to acknowledge the Skolkovo Foundation and BP for financial support. PA-A thanks the support of CONACYT Mexico for the award of his PhD grant. The Russian team would like to thank their based budget project V.47.1.3 (№ 0303-2014-0029)

\section{References}

[1] M. Watanabe, S.n. Kato, S. Ishizeki, H. Inomata, R.L. Smith Jr, Heavy oil upgrading in the presence of high density water: Basic study, Journal of Supercritical Fluids, 53 (2010) 48-52.

[2] M.S. Rana, V. Sámano, J. Ancheyta, J.A.I. Diaz, A review of recent advances on process technologies for upgrading of heavy oils and residua, Fuel, 86 (2007) 1216-1231.

[3] W.L. Nelson, ESTIMATING THE COST OF REPLACING A REFINERY, Oil and Gas Journal, 75 (1977) 81-

83.

[4] T. Ren, M. Patel, K. Blok, Olefins from conventional and heavy feedstocks: Energy use in steam cracking and alternative processes, Energy, 31 (2006) 425-451. 
[5] R.P. Dutta, W.C. McCaffrey, M.R. Gray, K. Muehlenbachs, Thermal cracking of Athabasca bitumen: Influence of steam on reaction chemistry, Energy and Fuels, 14 (2000) 671-676.

[6] V.I. Sharypov, B.N. Kuznetsov, N.G. Beregovtsova, S.V. Baryshnikov, V.N. Sidel'nikov, Steam cracking of coal-derived liquids and some aromatic compounds in the presence of haematite, Fuel, 75 (1996) 791-794.

[7] J.L. Pinilla, P. Arcelus-Arrillaga, H. Puron, M. Millan, Selective Catalytic Steam Cracking of anthracene using mesoporous Al $2 \mathrm{O} 3$ supported Ni-based catalysts doped with $\mathrm{Na}$, Ca or K, Applied Catalysis A: General, 459 (2013) 17-25.

[8] E. Fumoto, S. Sato, T. Takanohashi, Production of light oil by oxidative cracking of oil sand bitumen using iron oxide catalysts in a steam atmosphere, Energy and Fuels, 25 (2011) 524-527.

[9] M. Morimoto, Y. Sugimoto, Y. Saotome, S. Sato, T. Takanohashi, Effect of supercritical water on upgrading reaction of oil sand bitumen, Journal of Supercritical Fluids, 55 (2010) 223-231.

[10] R.O. Canıaz, C. Erkey, Process intensification for heavy oil upgrading using supercritical water, Chemical Engineering Research and Design, 92 (2014) 1845-1863.

[11] P.C. Mandal, M. Sasaki, M. Goto, Non-catalytic vanadium removal from vanadyl etioporphyrin (VOEP) using a mixed solvent of supercritical water and toluene: A kinetic study, Fuel, 92 (2012) 288-294.

[12] P.C. Mandal, M. Sasaki, M. Goto, Nickel removal from nickel etioporphyrin (Ni-EP) using supercritical water in the absence of catalyst, Fuel Processing Technology, 104 (2012) 67-72.

[13] A. Ates, G. Azimi, K.-H. Choi, W.H. Green, M.T. Timko, The role of catalyst in supercritical water desulfurization, Applied Catalysis B: Environmental, 147 (2014) 144-155.

[14] I. Kozhevnikov, A. Nuzhdin, O. Martyanov, Transformation of petroleum asphaltenes in supercritical water, The Journal of Supercritical Fluids, 55 (2010) 217-222.

[15] J. Towfighi, M. Sadrameli, A. Niaei, Coke Formation Mechanisms and Coke Inhibiting Methods in Pyrolysis Furnaces, Journal of chemical engineering of Japan, 35 (2002) 923-937.

[16] N. Li, B. Yan, X.-M. Xiao, A Review of Laboratory-Scale Research on Upgrading Heavy Oil in Supercritical Water, Energies, 8 (2015) 8962-8989.

[17] T.M. Yeh, J.G. Dickinson, A. Franck, S. Linic, L.T. Thompson, P.E. Savage, Hydrothermal catalytic production of fuels and chemicals from aquatic biomass, Journal of Chemical Technology and Biotechnology, 88 (2013) 13-24.

[18] P.E. Savage, A perspective on catalysis in sub-and supercritical water, The Journal of Supercritical Fluids, 47 (2009) 407-414.

[19] H. Weingärtner, E.U. Franck, Supercritical water as a solvent, Angewandte Chemie - International Edition, 44 (2005) 2672-2692.

[20] A. Kruse, H. Vogel, Heterogeneous Catalysis in Supercritical Media: 2. Near-Critical and Supercritical Water, Chemical engineering \& technology, 31 (2008) 1241-1245.

[21] E. Fumoto, T. Tago, T. Masuda, Production of lighter fuels by cracking petroleum residual oils with steam over zirconia-supporting iron oxide catalysts, Energy and Fuels, 20 (2006) 1-6.

[22] S. Funai, E. Fumoto, T. Tago, T. Masuda, Recovery of useful lighter fuels from petroleum residual oil by oxidative cracking with steam using iron oxide catalyst, Chemical Engineering Science, 65 (2010) 6065.

[23] E. Furimsky, Hydroprocessing in aqueous phase, Industrial \& Engineering Chemistry Research, 52 (2013) 17695-17713.

[24] M.T. Timko, A.F. Ghoniem, W.H. Green, Upgrading and desulfurization of heavy oils by supercritical water, The Journal of Supercritical Fluids, 96 (2015) 114-123.

[25] L.N. Han, R. Zhang, J.C. Bi, Reaction property of coal tar and its fractions in supercritical water, Ranliao Huaxue Xuebao/Journal of Fuel Chemistry and Technology, 36 (2008) 653-659.

[26] M. Jia, P. Afanasiev, M. Vrinat, The influence of preparation method on the properties of NiMo sulfide catalysts supported on ZrO2, Applied Catalysis A: General, 278 (2005) 213-221. 
Fuel, 182 (2016) 740-748, http://dx.doi.org/10.1016/j.fuel.2016.06.038

[27] M.A. Ermakova, D.Y. Ermakov, High-loaded nickel-silica catalysts for hydrogenation, prepared by sol-gel: Route - Structure and catalytic behavior, Applied Catalysis A: General, 245 (2003) 277-288.

[28] A. Karnaukhov, V. Fenelonov, V.Y. Gavrilov, Study of the effect of surface chemistry and adsorbent texture on adsorption isotherms by comparative method, Pure and Applied Chemistry, 61 (1989) 19131920.

[29] K.S. Sing, S. Gregg, Adsorption, surface area and porosity, Academic Press, London, (1982) 1-5. [30] J.L. Pinilla, A.B. García, K. Philippot, P. Lara, E.J. García-Suárez, M. Millan, Carbon-supported Pd nanoparticles as catalysts for anthracene hydrogenation, Fuel, 116 (2014) 729-735.

[31] L. Qu, W. Zhang, P.J. Kooyman, R. Prins, MAS NMR, TPR, and TEM studies of the interaction of NiMo with alumina and silica-alumina supports, Journal of Catalysis, 215 (2003) 7-13.

[32] P. Arnoldy, J.C.M. De Jonge, J.A. Moulijn, Temperature-programmed reduction of $\mathrm{MoO} 3$ and $\mathrm{MoO} 2$, Journal of Physical Chemistry, 89 (1985) 4517-4526.

[33] M. Henker, K.P. Wendlandt, J. Valyon, P. Bornmann, Structure of MoO3/Al2O3-SiO2 catalysts, Applied Catalysis, 69 (1991) 205-220.

[34] S. Rajagopal, H.J. Marini, J.A. Marzari, R. Miranda, Silica-Alumina-Supported Acidic Molybdenum Catalysts - TPR and XRD Characterization, Journal of Catalysis, 147 (1994) 417-428.

[35] J.L. Brito, J. Laine, K.C. Pratt, Temperature-programmed reduction of Ni-Mo oxides, Journal of Materials Science, 24 (1989) 425-431.

[36] J.A. Marzari, S. Rajagopal, R. Miranda, Bifunctional Mechanism of Pyridine Hydrodenitrogenation, Journal of Catalysis, 156 (1995) 255-264.

[37] Y. Kida, C.A. Class, A.J. Concepcion, M.T. Timko, W.H. Green, Combining experiment and theory to elucidate the role of supercritical water in sulfide decomposition, Physical Chemistry Chemical Physics, 16 (2014) 9220-9228.

[38] Z.-M. Cheng, Y. Ding, L.-Q. Zhao, P.-Q. Yuan, W.-K. Yuan, Effects of supercritical water in vacuum residue upgrading, Energy \& fuels, 23 (2009) 3178-3183.

[39] M. Daud, J.L. Pinilla, P. Arcelus-Arrillaga, K. Hellgardt, R. Kandiyoti, M. Millan, Heavy oil upgrading in subcritical and supercritical water: Studies on model compounds, in: ABSTRACTS OF PAPERS OF THE AMERICAN CHEMICAL SOCIETY, AMER CHEMICAL SOC 1155 16TH ST, NW, WASHINGTON, DC 20036 USA, 2012.

[40] J. DeCoster, A. Ergut, Y.A. Levendis, H. Richter, J.B. Howard, J.B. Carlson, PAH emissions from hightemperature oxidation of vaporized anthracene, Proceedings of the Combustion Institute, 31 I (2007) 491-499.

[41] L.Q. Zhao, Z.M. Cheng, Y. Ding, P.Q. Yuan, S.X. Lu, W.K. Yuan, Experimental study on vacuum residuum upgrading through pyrolysis in supercritical water, Energy and Fuels, 20 (2006) 2067-2071.

[42] L. Pastor-Pérez, R. Buitrago-Sierra, A. Sepúlveda-Escribano, CeO2-promoted Ni/activated carbon catalysts for the water-gas shift (WGS) reaction, International Journal of Hydrogen Energy, 39 (2014) 17589-17599.

[43] L. Pastor-Pérez, T.R. Reina, S. Ivanova, M.Á. Centeno, J.A. Odriozola, A. Sepúlveda-Escribano, NiCeO2/C catalysts with enhanced OSC for the WGS reaction, Catalysts, 5 (2015) 298-309.

[44] S.D. Senanayake, J. Evans, S. Agnoli, L. Barrio, T.L. Chen, J. Hrbek, J.A. Rodriguez, Water-gas shift and $\mathrm{CO}$ methanation reactions over Ni-CeO2(111) catalysts, Topics in Catalysis, 54 (2011) 34-41. 